\title{
PARTIAL CRICOIDECTOMY WITH PRIMARY THYROTRACHEAL ANASTOMOSIS FOR POSTINTUBATION SUBGLOTTIC STENOSIS
}

Paolo Macchiarini, $\mathrm{MD}, \mathrm{PhD}^{\mathrm{a}}$

Jean-Philippe Verhoye, $\mathrm{MD}^{\mathrm{b}}$

Alain Chapelier, $\mathrm{MD}, \mathrm{PhD}^{\mathrm{b}}$

Elie Fadel, MD ${ }^{\mathrm{b}}$

Philippe Dartevelle, $\mathrm{MD}^{\mathrm{b}}$
Objective: We describe a Pearson-type technique and evaluate its results for postintubation subglottic stenosis.

Methods: Forty-five patients underwent a partial cricoidectomy with primary thyrotracheal anastomosis, and 5 underwent simultaneous repair of a tracheoesophageal fistula as well. Twenty-four (53\%) patients were referred to us after initial conservative $(n=21)$ or operative $(n=3)$ management. There were 27 cuff lesions, 7 stomal lesions, and 11 at both levels. The upper limit of the stenosis was $1.5 \mathrm{~cm}$ (range, $1-2.5 \mathrm{~cm}$ ) below the cords, and the subglottic diameter was reduced by $60 \%$ in $38(84 \%)$ of the patients. The length of airway resection ranged from 2 to $6 \mathrm{~cm}$ (median, $3 \mathrm{~cm}$ ). Despite 23 thyrohyoid or suprahyoid releases, 8 anastomoses were under tension.

Results: Thirty-seven ( $82 \%)$ patients were extubated after the operation $(n=30)$ or within 24 hours $(n=7)$. Six patients required postoperative airway stenting (median, 5.5 days). Early ( $<30$ days) complications occurred in $18(41 \%)$ patients, mainly as transient airway and voice complaints, aspiration, and dysphagia. One (2\%) patient died of myocardial infarction. Late morbidities were 2 failures occurring as bilateral recurrent nerve paralysis and restenosis requiring definitive tracheostomy. Patients had excellent or good anatomic $(n=42[96 \%])$, functional $(n=41[93 \%])$, or both types of long-lasting results, with no stenotic relapse.

Conclusions: Partial cricoidectomy with primary thyrotracheal anastomosis can be applied in patients with postintubation stenosis extending up to $1 \mathrm{~cm}$ below the cords and measuring up to $6 \mathrm{~cm}$ in length with excellent-to-good definitive results. The association with a tracheoesophageal fistula does not contraindicate surgical repair. (J Thorac Cardiovasc Surg 2001;121:68-76)
S tenosis of the upper airway lying between the vocal $\mathcal{O}$ cords superiorly and cricoid cartilage and uppermost cervical trachea below may be of different origin but most frequently are due to mechanical ventilation. ${ }^{1}$ Their most common genesis is pressure necrosis by the endotracheal tube, tracheostomy tube, or, more often, their attached (overinflated) cuffs (Fig 1). This usually results in circumferential erosion evolving in varying

From the Departments of Thoracic and Vascular Surgery, ${ }^{\mathrm{a}}$ Heidehaus Hospital, Hannover Medical School, Hannover, Germany, and Thoracic and Vascular Surgery and Heart-Lung Transplantation, ${ }^{\mathrm{b}}$ Hôpital Marie-Lannelongue, Le Plessis Robinson, Paris-Sud University, France.

Read at the Eightieth Annual Meeting of The American Association for Thoracic Surgery, Toronto, Ontario, Canada, April 30-May 3, 2000.

Received for publication May 4, 2000; revisions requested Aug 9, 2000; revisions received Aug 25, 2000; accepted for publication Sept 8, 2000. degrees of stenosis, especially at the narrowest part of the airway, the cricoid cartilage.

In 1975, Pearson and colleagues ${ }^{2}$ described an original technique that removes the anterior arch of the cricoid cartilage, preserves the posterior plate of the cricoid to protect the recurrent laryngeal nerves, and advances healthy tracheal tissue to reconstruct the airway directly. In his and other ${ }^{1-4}$ studies, its success rate

\footnotetext{
Address for reprints: Paolo Macchiarini, MD, PhD, Department of Thoracic and Vascular Surgery, Heidehaus Hospital, Hannover Medical School, Am Leineufer, 70, 30419 Hannover, Germany (E-mail: pmacchiarini@compuserve.com).

Copyright () 2001 by The American Association for Thoracic Surgery

$0022-5223 / 2001 \$ 35.00+0 \quad \mathbf{1 2 / 6 / 1 1 1 4 2 0}$
}

doi:10.1067/mtc.2001.111420 
Table I. Demographic profiles of patients

\begin{tabular}{ll}
\hline Characteristics & No. $(n=45)$ \\
\hline Age, y (median [range]) & $42(4-77)$ \\
Sex (M/F) & $31(66 \%) / 14(34 \%)$ \\
Cause of mechanical ventilation & \\
$\quad$ Medical conditions & $13(29 \%)$ \\
$\quad$ Postoperative complications & $12(27 \%)$ \\
Post-traumatic & $17(38 \%)$ \\
$\quad$ Suicide & $3(6 \%)$ \\
Tracheotomy (present/absent) & $24(53 \%) / 21(47 \%)$ \\
\hline
\end{tabular}

Table II. Anatomic characteristics of the subglottic stenosis observed at preoperative bronchoscopy

\begin{tabular}{ll}
\hline Characteristics & No. $(n=45)$ \\
\hline Distance from vocal cords $(\mathrm{cm})$ & $1.5(1-2.5)$ \\
Length subglottic stenosis $(\mathrm{cm})$ & $2(1-5)$ \\
Distance from carina $(\mathrm{cm})$ & $6(5-7.5)$ \\
Length of TEF $(\mathrm{n}=5 ; \mathrm{cm})$ & $2.5(1-3.5)$ \\
\hline
\end{tabular}

Data are expressed as median (range)

has been much higher than most of the more complex procedures both in children and adults. This article describes a Pearson-type partial cricoidectomy with primary thyrotracheal anastomosis and updates our early ${ }^{5}$ experience with this technique for postintubation subglottic stenosis.

\section{Patients and methods}

A total of 115 patients with postintubation stenosis of the subglottic, cervical, or intrathoracic airway were operated on since January 1981. Among 73 patients whose stenosis was preoperatively supposed to involve only the cervical or intrathoracic trachea, 5 (7\%) required a subglottic resection, whereas $2(5 \%)$ of the 42 patients undergoing subglottic stenosis received a simple tracheal resection. Therefore, there was a total of 70 tracheal and 45 subglottic resections, with the last being the subject of this report.

All patients underwent (1) an anatomic evaluation of the exact location and extent of subglottic and tracheal involvement and state of the cricoid plate and (2) a functional evaluation of the glottic opening, vocal cord mobility and trophicity, and recurrent nerve function by routine radiology with fluoroscopy, tracheal tomograms, laryngotracheal computed tomography, and examination with a flexible or rigid bronchoscope. All other preoperative examinations were those performed as in any tracheal operation.

Patient characteristics. Patient profile is depicted in Table I. Five $(11 \%)$ were associated with a laryngopharynx $(n=1)$ or tracheoesophageal $(n=4)$ fistula (TEF). All but 4 patients were operated on at the Marie Lannelongue Hospital. Two patients initially had an emergency tracheotomy, 4 had a nasotracheal and 39 an orotracheal tube as the initial site of

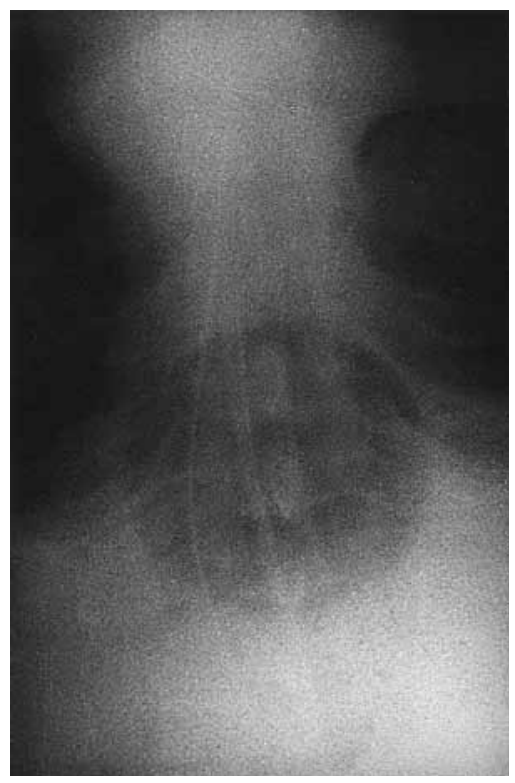

Fig 1. Chest radiograph of a patient with a postintubation subglottic stenosis showing an extremely overinflated cuff of the orotracheal tube, a usually common scenario in the histories of these patients.

intubation, and the overall median intubation time was 21 days (range, 5-60 days). Overall, the median diagnostic delay after the initial intubation was 30 days (range, 0-425 days), and diagnosis occurred either before $(\mathrm{n}=3[7 \%])$, at $(\mathrm{n}=15$ [33\%]), or after $(\mathrm{n}=27[60 \%])$ extubation. Twenty $(44 \%)$ patients presented with dyspnea and stridor at rest on arrival. Among the 24 patients who had a tracheotomy in their history, the tracheostomy site was usually between the first and second tracheal rings in the vast majority and was normally scarred in 12 patients, infected in 3 patients, inflammatory in 7 patients, and ischemic in 2 patients. None of the tracheotomies were translaryngeal.

Twenty-four $(53 \%)$ patients underwent prior conservative $(\mathrm{n}=21)$ or operative $(\mathrm{n}=3)$ attempts to correct their stenosis elsewhere, including multiple laser treatments $(\mathrm{n}=19)$ with $(\mathrm{n}=7)$ and without $(\mathrm{n}=12)$ interval dilations, placement of a Montgomery T tube $(\mathrm{n}=2)$, segmental resection, and tracheotracheal anastomosis $(\mathrm{n}=2)$ and the Rethi ${ }^{6}$ procedure $(\mathrm{n}=1)$. One patient had unilateral vocal cord paralysis, and 3 patients had complete loss of phonation preoperatively. One patient had a right single lung transplantation 4 years before. Twenty patients had steroid treatments before arrival.

Subglottic stenosis profile and TEF management. Table II outlines the preoperative anatomic characteristics of the subglottic stenoses. There were 27 cuff-level lesions, 7 stomal injuries, and 11 at both levels. The subglottic normal crosssectional area was reduced by at least $60 \%$ in $38(84 \%)$ of the patients. The upper limit of the stenoses was between 1 and 2 $\mathrm{cm}$ below the vocal cords in 33 (73\%) patients, and the medi- 


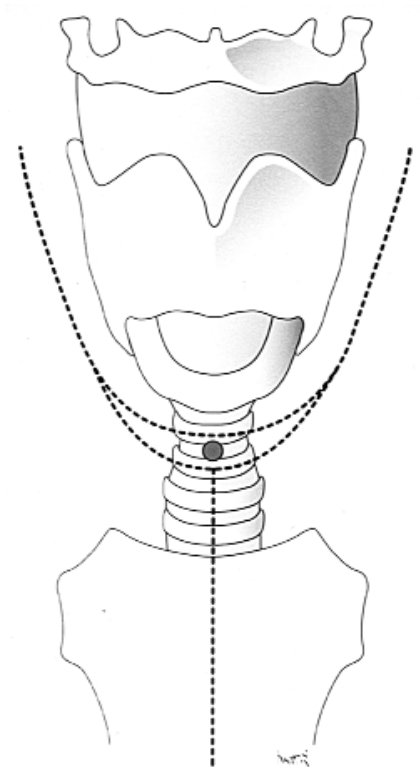

Fig 2. Diagram showing the U-shaped cervical incision used to approach subglottic resections. Inferiorly, the incision can be prolonged vertically just below the manubrial notch to include an upper median sternotomy. The management of the tracheal stoma is done as in other tracheal operations.

an overall endoscopic length of the stenoses was $2 \mathrm{~cm}$ (range, $1-5 \mathrm{~cm})$. The subglottic stenosis was circular in 38 (84\%) and near-complete in 4 patients; $3(6 \%)$ patients had a second stenotic process located $1.5 \pm 0.4 \mathrm{~cm}$ from the lower limit of the subglottic stenosis. An extensive tracheomalacia was associated in 2 patients.

The preoperative management of patients having a subglottic stenosis and a TEF included stabilization with adequate nutritional provision and complete infectious control, removal of the indwelling nasogastric tube, placement of a new low-pressure high-volume tracheostomy tube with a cuff below the site of the fistula, and positioning of draining gastrostomy and feeding jejunostomy tubes. ${ }^{6}$ Patients with local signs of infection or inflammation were usually managed with antibiotics with or without bougie dilations or the placement of a tracheostomy tube below the subglottic stenosis.

Anesthesia. The same principles used for any airway operation were systematically used. To allow spontaneous ventilation during operation and early extubation, continuous infusion techniques with short-acting muscle relaxants and rapidly metabolized intravenous anesthetic agents and shortacting opioids were used. Intubation was usually done through an endotracheal tube placed just above the lesion (for lesions $>6 \mathrm{~mm}$ in diameter) or the tracheostomy, inducing spontaneous ventilation. When necessary (for lesions $<6 \mathrm{~mm}$ in diameter), the airway was dilated immediately before operations, with the patient asleep, by using either the laryngoscope or a gum-tipped esophageal bougie. By following these simple atraumatic techniques, a $6.5 \mathrm{~F}$ uncuffed endotracheal

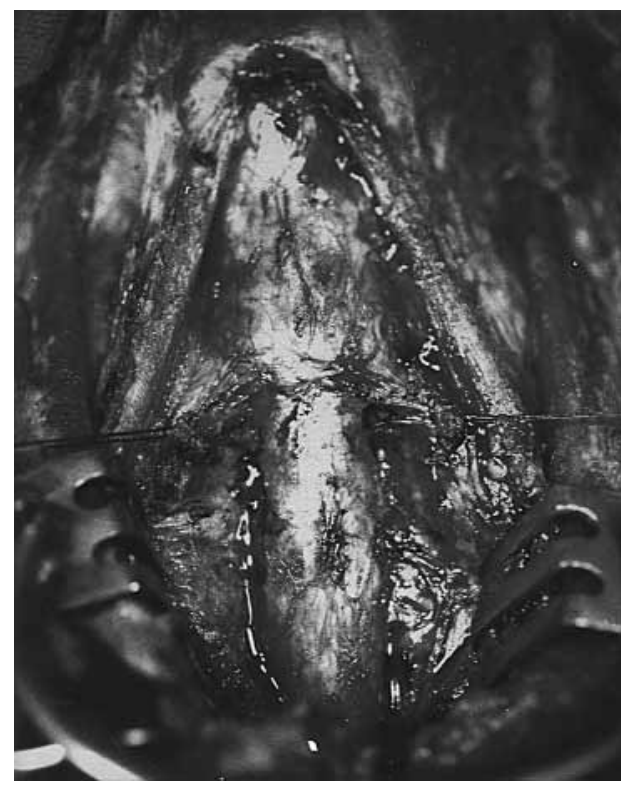

Fig 3. Operative view after careful vascular-sparing dissection of the airway from the level of the inferior border of the cricoid ring above and the inferior limit of the stenosis below. Dissection is maintained against the outer surface of the airway to protect the recurrent laryngeal nerves, which are frequently obscured by peritracheal dense scarring.

tube can usually be passed beyond the stenosis. We do not favor a rigid bronchoscope as a dilating instrument because of its more traumatic local effects.

Once the distal airway was divided, ventilation was obtained by inserting an armored endotracheal tube in the distal tracheal segment by using sterile connections across the operative field. Over the years, we found it simpler to use intermittent cross-field intubation with an armored tube for resection of the upper airway than jet ventilation.

Operative procedure. Only those patients whose stenosis was not associated with local infection or inflammation and those not receiving steroid $(<10-15 \mathrm{mg} / \mathrm{d})$ treatment were operated on immediately. Our surgical technique ${ }^{5}$ basically reflects the principles described by Gerwat and Bryce ${ }^{8}$ and Pearson and colleagues, ${ }^{2}$ with a few modifications.

The patient is placed in a supine position with a bag beneath the shoulders and the head hyperextended. Through an anterior cervical U-shaped incision (Fig 2), eventually including the tracheostomy stoma if present, the skin, subcutaneous fat, and platysma are elevated as one layer, and the flap is raised to the level of the suprahyoid region. Inferiorly, an extension vertical incision is made to the manubrial notch. The sternohyoid muscles are divided in the midline, and the thyroid isthmus is divided and ligated to expose the anterior surface of the cervical trachea. The trachea is freed circumferentially to the level of the inferior border of the cricoid cartilage above and the inferior limit of the stenosis below. This stage of the operation is completed when the anterior and lateral surfaces of the thyroid cartilage, the cricothyroid muscle, 


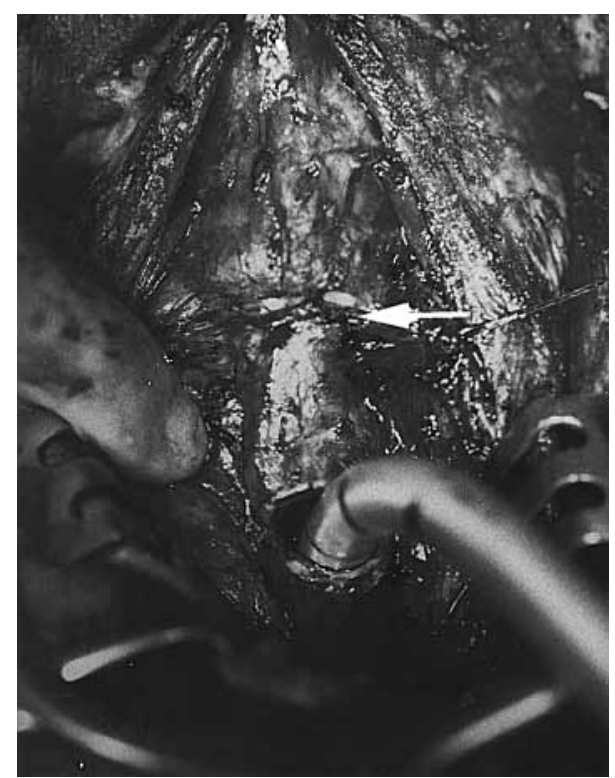

Fig 4. Operative view showing division of the trachea below the damaged area and distal ventilation through insertion of an armored endotracheal tube in the distal tracheal segment by using sterile connections across the operative field. Arrow indicates inferior border of the cricoid arch.

the cricoid cartilage, and the cervical trachea are exposed (Fig 3 ). We usually do not expose and incise the perichondrium over the cricoid arch or cartilage.

Special care is taken to maintain the dissection close to the outer tracheal surface to avoid injury to both recurrent laryngeal nerves, but no attempt is made to identify the recurrent nerves because they are almost obscured by peritracheal scarring. Dissection should be done carefully to preserve the lateral blood supply to the unresected trachea. The trachea is first divided either at the midpoint or below (Fig 4) the damaged area, and 2 lateral traction sutures are placed in the distal tracheal wall. It is of paramount importance to ensure that the tracheal cut ends have a normal diameter and are as healthy as possible. Dissection of the posterior wall of the trachea from the esophagus is then performed from the caudal area to the upper limit of the damaged area.

The subglottic larynx is usually resected, without following a subperichondrial plane, through an oblique $\left(30^{\circ}-40^{\circ}\right.$ with respect to the larynx axis) incision starting anteriorly either at the inferior border of the thyroid cartilage or the superior border of the cricoid arch and extending posteriorly and inferiorly through the lower margin of the cricoid plate below the point of entry of the recurrent laryngeal nerves (Fig 5). This oblique division obtains a complete resection of the anterior cricoid arch and opens at maximum the airway below the vocal cords (Fig 6). A higher level of subperichondrial transection posteriorly up to a few millimeters of the inferior margins of the vocal folds may be obtained by removing, with fine rongeurs, the anterior
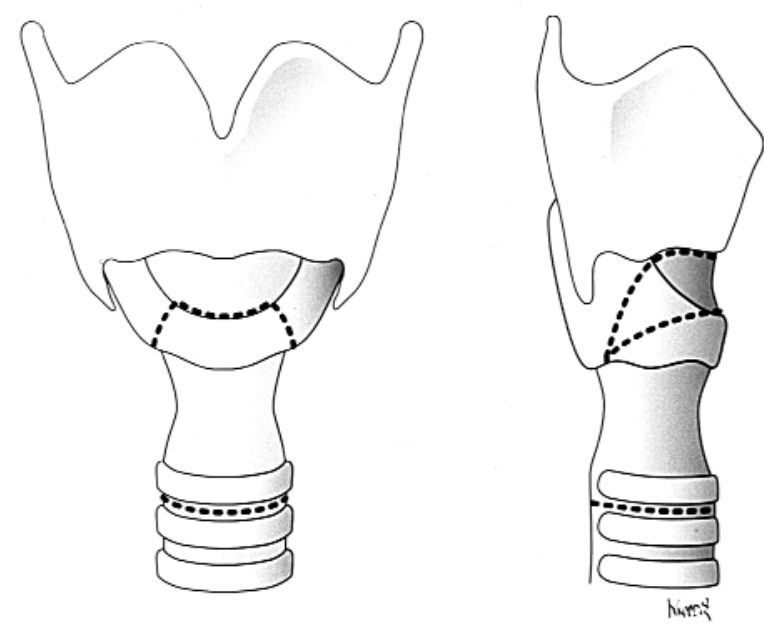

Fig 5. Diagram showing the lines of proximal and distal transections. The proximal division starts anteriorly either at the inferior border of the thyroid cartilage or the superior border of the cricoid arch, depending on the distance between the stenosis and the vocal cords. It extends posteriorly and inferiorly through the lower margin of the cricoid plate below the point of entry of the recurrent laryngeal nerves.

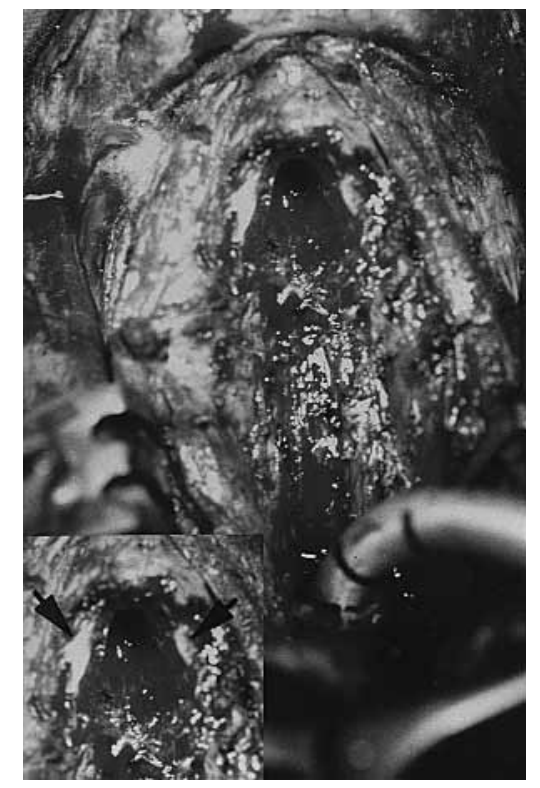

Fig 6. Operative view proximally showing the complete resection of the anterior cricoid arch and distally showing the distal ventilation through an armored endotracheal tube. The resection of the anterior cricoid was not subperichondrial, and this is highlighted in the insert. This $30^{\circ}$ to $40^{\circ}$ oblique cricoidectomy gives a maximal overture at the subglottic level, where the thyrotracheal anastomosis can be made safely. 

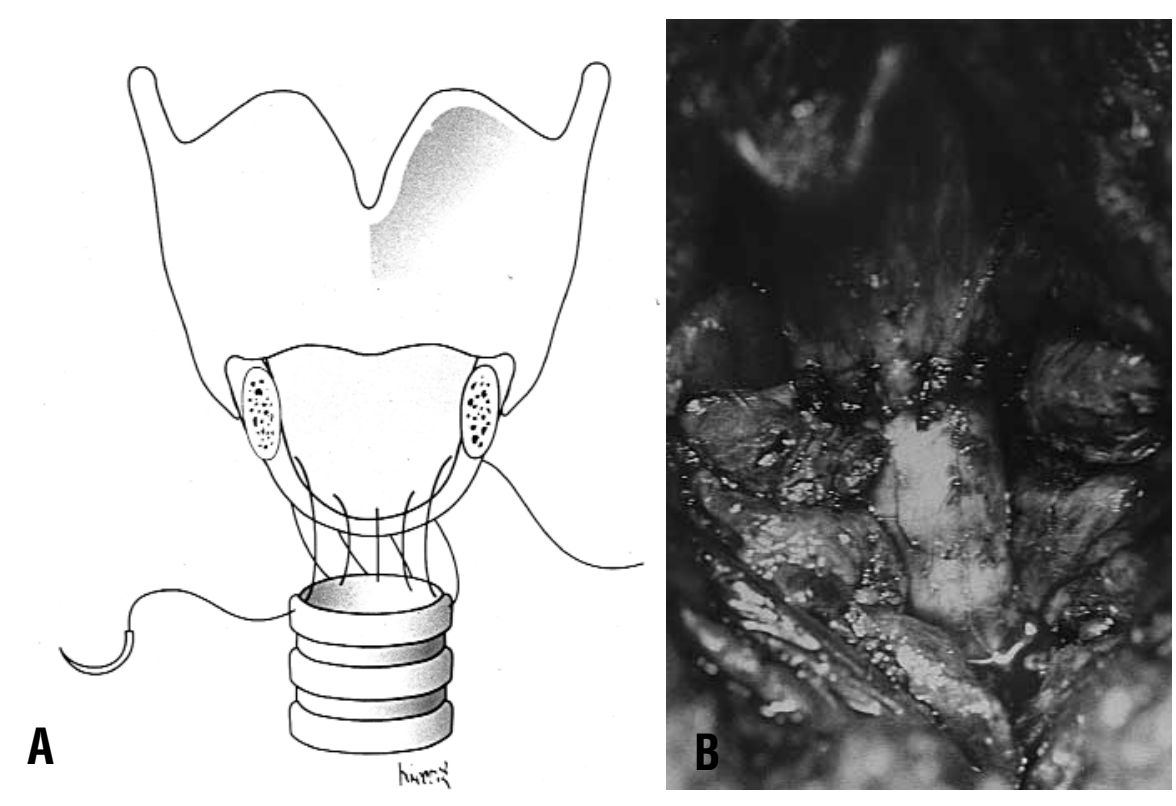

Fig 7. A, Diagram showing the posterior continuous 4-0 polydioxanone suture placed between the remnants of the posterior mucosa (proximally) and the healthy trachea (distally). This running suture usually picks up the residual healthy mucosa. For very proximal stenoses, we do use this suture, even on the diseased mucosa, high in the posterior larynx because the big cross-sectional cricotracheal diameter resulting from the oblique incision allows the regression of the inflammatory mucosal phenomena and has no long-term sequelae. One might, however, avoid using the running suture on the bare posterior cricoid plate, and the posterior flap, as described by Grillo and colleagues ${ }^{1}$ would then be the solution. B, Operative view after completion of the anterior anastomosis made here between the uppermost tracheal cartilage and lower margin of the thyroid cartilage with interrupted 3-0 polyglactin sutures, the knots of which are tied outside. When present, the air leakages are usually seen in the middle of the anterior anastomosis, are minimal, and are treatable conservatively by simply opening the tissue layers overlying the air leakage.

aspect of the remaining posterior cricoid plate, following a submucosal plane because this and its chorion represent a formidable solid plane where sutures hold very well. Before the complete transection of the airway, the original orotracheal tube is withdrawn above the level of the vocal cords by the anesthetist, and its tip is secured with silk sutures, so that it may be guided back through the glottis when the anastomosis is being completed. Sometimes, when the original orotracheal tube cannot be passed at this stage, it is possible to pass a sterile orotracheal tube retrograde from below without injuring the cords.

The primary anastomosis is then achieved, without plicating the distal membranous cervical trachea, with a posterior cricotracheal anastomosis by using a continuous 4-0 polydioxanone (PDS; Ethicon, Inc, Somerville, NJ) suture (Fig 7, $A$ ) and an anterior thyrotracheal anastomosis by using interrupted 3-0 polyglactin (Vicryl; Ethicon, Inc) sutures placed between the uppermost tracheal cartilage and the cricothyroid membrane or the lower margin of the thyroid cartilage, the knots of which are tied outside (Fig 7, B). To avoid excessive tension of the anastomosis, various techniques of tracheal mobilization and laryngeal release may be necessary.

In patients with a $\mathrm{TEF},{ }^{7}$ the recurrent laryngeal nerve or nerves should be exposed, but not dissected, in the tracheo- esophageal groove and identified at a location remote from the fistula. Identification of the fistulous tract may not be simple and requires a more extensive dissection than expected. Once the fistulous tract is dissected and individualized, it is divided. The esophageal defect is then closed by using a 2layer mucosal and muscular closure after debridement of the fistulous edges with interrupted 3-0 Vicryl sutures. Because of the reduced airway but unchanged esophageal length, the esophageal and tracheal suture lines are not at all in contact with each other, and eventual muscle interpositions or esophageal rotations are unnecessary.

The wound is then filled with sterile saline solution to test the anastomosis. The cervical incision is closed by covering the anastomotic line with the thyroid isthmus and approaching the linea alba cervicalis. Two soft, multiholed drain catheters are placed. The mobility of the vocal cords is then assessed, and, depending on the adequacy of the airway immediately after operation and absence of bilateral recurrent nerve palsy, the patient is extubated immediately postoperatively. Protective distal tracheostomy is usually not performed.

Follow-up studies. All patients were observed until death or last date of follow-up (March 1, 2000). None of them were lost to follow-up, and the efficacy of the operations was eval- 
Table III. Early (<30 days) surgical complications in surviving patients $(n=44)$

\begin{tabular}{lc}
\hline Types & No. \\
\hline Airway & \\
$\quad$ Air leak & $3(7 \%)$ \\
Glottic edema & $4(9 \%)$ \\
Granuloma & $10(23 \%)$ \\
Restenosis & $2(4 \%)$ \\
Voice & \\
Hoarse & $18(41 \%)$ \\
Weak & $15(23 \%)$ \\
Others & \\
Wound infection & $2(4 \%)$ \\
Aspiration & $10(23 \%)$ \\
Delayed stomal closure & $2(4 \%)$ \\
Dysphagia & $9(20 \%)$ \\
\hline
\end{tabular}

${ }^{*}$ Clinically and radiologically documented.

uated by means of clinical and investigational examinations in all surviving patients. The anatomic and functional criteria mentioned above were used postoperatively to assess outcome that was expressed as excellent (without any sequelae), good (minor sequelae not affecting quality of life), satisfactory (patients are stressed on exercise, with abnormal vocal cords and narrowed anastomosis), or failure, both anatomically and functionally. ${ }^{7}$

\section{Results}

The vast majority of the operations were done on a (nearly) emergency basis, whereas all patients who had a TEF underwent the above-mentioned preoperative management and elective operations after a median of 25 days (range, 5-45 days). Only 3 patients were intraoperatively ventilated through the tracheostomy tube because the stenosis totally obstructed the subglottic airway.

Surgical characteristics. All but one (manubriotomy) operation was done through a cervical U-shaped incision. The median length of resected airway was 3 $\mathrm{cm}$ (range, $2-6 \mathrm{~cm}$ ) overall ( $<2 \mathrm{~cm}$ in 12 patients, $2.5-4$ $\mathrm{cm}$ in 23 patients, and $4-6 \mathrm{~cm}$ in 10 patients). Although $23(51 \%)$ thyrohyoid $(\mathrm{n}=19)$ or suprahyoid $(\mathrm{n}=4)$ releases reduced anastomotic tension, 8 (18\%) anastomoses were under some degree of tension. This happened more frequently in patients having more than 4 $\mathrm{cm}$ of airway resected $(\mathrm{n}=7$ [70\%]) than in those with less airway resection $(\mathrm{n}=1[3 \%], P=.0001)$. The tracheostomy stoma was excised in 17 , closed in 4 , and used in 3 patients. Because of the scarred nature of the subglottic mucosa above the line of transection, 1 patient had a vertical section of the posterior cricoid plate with the interposition of autogenous cartilage, and 2 patients had a subtotal cricoid plate resection (massive chondritis).

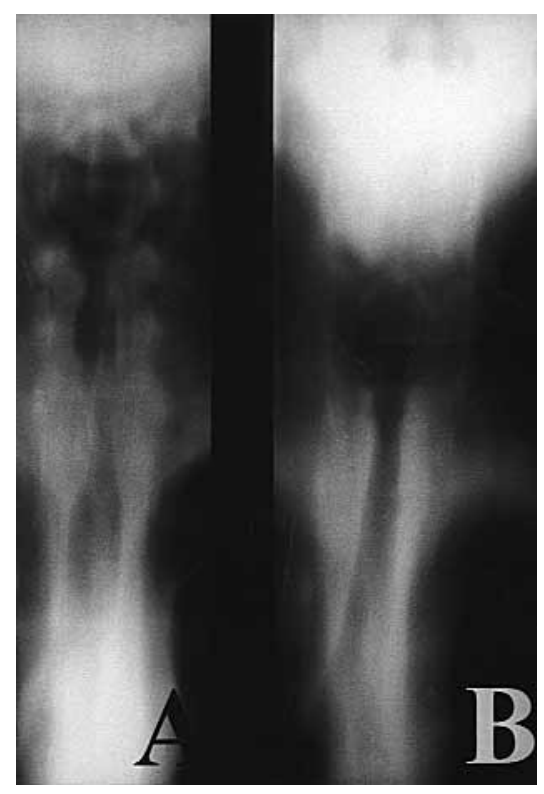

Fig 8. Preoperative (A) and postoperative (B) operative tomograms of a patient undergoing a subglottic resection and anastomosis. A, The stenosis was $1.5 \mathrm{~cm}$ below the vocal cord. B, The postoperative tomogram shows the direct anastomosis between the uppermost healthy trachea and the thyroid cartilage, as per Fig 7, B.

Thirty-seven $(82 \%)$ patients were extubated immediately after the operation $(n=30)$ or within 24 hours $(n=7)$. Because of the extensive airway resection, subtotal plate resection, double laryngopharyngeal fistula and $\mathrm{TEF}$, and surgical failure, an intra-airway stenting in the form of a single-lumen orotracheal tube was left in place in 6 patients (all but 1 with a subglottic stenosis only) for a median of 5.5 days (range, 4-65 days).

Morbidity and mortality. One $(2 \%)$ postoperative (day 1) death occurred after an acute myocardial infarction without evidence of subglottic problems. Eighteen $(41 \%)$ patients had early ( $<30$ days) complications, mainly as airway and voice complaints, aspiration, and dysphagia (Table III), and all but one restenosis requiring definitive tracheotomy were successfully managed conservatively or surgically (one reoperation for a restenosis and all wound infections). As expected, the deglutition disturbances were significantly $(P=.02)$ higher in patients with a TEF $(\mathrm{n}=4[80 \%])$ than in those without $(\mathrm{n}=6[18 \%])$, but no other outcome differences were observed between the 2 pathologies. Late morbidities ( $>30$ days) were a definitive bilateral recurrent nerve paralysis and the previously mentioned restenosis occurring in a 23-year-old woman in whom the proximal anastomosis, made in a 
Table IV. Long-term anatomic and functional results of subglottic resection and reconstruction for the 44 surviving patients with postintubation stenosis

\begin{tabular}{|c|c|c|c|c|c|c|c|c|}
\hline \multirow[b]{2}{*}{ Subglottic resection } & \multicolumn{2}{|c|}{ Excellent } & \multicolumn{2}{|c|}{ Good } & \multicolumn{2}{|c|}{ Satisfactory } & \multicolumn{2}{|c|}{ Failure } \\
\hline & $A$ & $F$ & $A$ & $F$ & $A$ & $F$ & $A$ & $F$ \\
\hline Without TEF $(n=39)$ & 30 & 32 & 7 & 5 & - & 1 & 2 & 2 \\
\hline With TEF $(\mathrm{n}=5)$ & 5 & 4 & - & - & - & 1 & - & - \\
\hline Total $(\mathrm{n}=44)$ & $35(80 \%)$ & $36(81 \%)$ & $7(16 \%)$ & $5(11 \%)$ & - & $2(4 \%)$ & $2(4 \%)$ & $2(4 \%)$ \\
\hline
\end{tabular}

$A$, Anatomic; $F$, functional.

Table V. Worldwide experience with subglottic resection and anastomosis for postintubation ${ }^{*}$ stenosis

\begin{tabular}{lccccc}
\hline Authors (references) & Year & No. of patients & Deaths & Good & Failed \\
\hline Ogura and Roper $^{10}$ & 1972 & 17 & 0 & 14 & 3 \\
Gerwat and Bryce $^{8}$ & 1974 & 4 & 0 & 4 & 0 \\
Maddaus and colleagues $^{11}$ & 1992 & 38 & 0 & 36 & 2 \\
Couraud and colleagues $^{3}$ & 1988 & 27 & 0 & 27 & 0 \\
Grillo and colleagues $^{1}$ & 1992 & 80 & 1 & 77 & 2 \\
Monnier and colleagues $^{4}$ & 1999 & 38 & 1 & 42 & 1 \\
Present series & 2000 & 45 & $2(1 \%)$ & $237(95 \%)$ & $12(4 \%)$ \\
Total & $1972-2000$ & 249 & & & 2 \\
\end{tabular}

*Some series include inflammatory subglottic lesions resulting from causes other than mechanical ventilation with intra-airway intubation.

pathologic area, contributed to the development of a subglottic and glottic restenosis requiring definitive tracheostomy above the second tracheal ring.

Long-term anatomic and functional results. The median hospitalization time was 19 days (range, 4-70 days). All surviving patients are alive after a median follow-up time of 9.6 years, and the majority of patients had excellent or good anatomic ( $\mathrm{n}=42$ [95\%]), functional $(\mathrm{n}=41$ [93\%]), or both types of long-lasting results (Table IV and Fig 8). There was no relapse of the stenosis during subsequent follow-up periods.

\section{Discussion}

Complete transection of the subglottic airway at any level above the cricothyroid joints divides the recurrent nerves, collapses the subglottis, and injures the vocal cords and would therefore be an inappropriate operation for subglottic strictures. Unsurprisingly, earlier attempts with subperichondrial cricoid resection, ${ }^{9}$ vertical bisection of the posterior cricoid lamina of the cricoid plate, ${ }^{6}$ segmental resection of the cricoid cartilage with primary thryrotracheal anastomosis, ${ }^{10}$ or partial oblique resection of the subglottic airway ${ }^{8}$ reported variable and unpredictable results. In 1975, Pearson and colleagues ${ }^{2}$ described a simple 1-stage technique that allowed transverse resection of subglottic strictures at any level below the vocal cords with preserva- tion of recurrent nerve function and airway stability through (1) a subperichondrial complete resection of the anterior cricoid arch and partial resection of the posterior cricoid plate and (2) a thyrotracheal (anteriorly) and cricotracheal (posteriorly) anastomosis. Because this technique treats all lesions extending up to $1 \mathrm{~cm}$ from the vocal cords, it shortly became the gold standard for resecting all subglottic stenoses, both in children and adults (Table V).

Our technique incorporates the principles of segmental resection of the cricoid cartilage with a primary thryrotracheal anastomosis described by Gerwat and Bryce ${ }^{8}$ in 1974 and differs from that of Pearson and colleagues ${ }^{2}$ in that it does not (routinely) resect subperichondrially the cricoid arch and plate. Our technique also differs from that of Grillo and colleagues ${ }^{1}$ in that we do not use a membraneous tracheal wall flap to resurface the posterior cartilage. It is therefore more of a variant, using some of the technical aspects of each of the above-mentioned techniques. The fact that more than $95 \%$ of our patients had satisfactory-to-excellent anatomic and functional results confirms our initial report on the efficacy of our partial cricoidectomy technique. ${ }^{5}$ The specific lessons learned over the years with this operation for subglottic postintubation stenosis are as follows.

First, any surgical attempt should be avoided until all inflammatory or infectious phenomena have vanished. 
Second, because the anastomosis must be performed in healthy tissue, it is imperative to delineate preoperatively the exact location and extent of the stenosis. The two failures we observed early in our experience were related to the extension of the stenosis into the glottic region, and because at that time we were unaware of the pioneering works by the Toronto group ${ }^{11,12}$ and Couraud and colleagues, ${ }^{13}$ the anastomoses were made in unhealthy tissue and without synchronous laryngeal reconstruction.

Third, the combination of the oblique transection made at $30^{\circ}$ to $40^{\circ}$ with respect to the laryngeal axis and the anterior arch cricoidectomy opens the subglottic airway maximally, where a safe reconstructive anastomosis can be made. Because the distal cervical trachea has a wide cross-sectional diameter, its anastomosis with the thyrocricoid membrane or thyroid cartilage opens the subglottic lumen in as much as 2 or 3 times that observed preoperatively in patients with postintubation subglottic stenosis.

Fourth, the importance of leaving an adequate rigid posterior cricoid support to the arytenoids cannot be underestimated, as reported by others. ${ }^{1-4}$ This is particularly true with the oblique cricoidectomy because with the anterior arch being removed, the larynx is toppled over as it loses its anterior rigid support, especially when longer tracheal resections are needed. Whenever a (sub)total posterior cricoid plate resection is necessary, then stabilization of the upper airway through endoluminal tubes until consolidation is mandatory to avoid laryngeal collapse and dysfunction. To avoid the tailoring of $\mathrm{T}$ tubes, we prefer to leave a single-lumen orotracheal tube across the anastomosis until necessary.

Fifth, the length of the stenosis itself should be less than $6 \mathrm{~cm}$, a basic principle of any tracheal operation. In our experience those resections longer than 4 to $5 \mathrm{~cm}$ were associated, despite the performance of release maneuvers, with a significantly higher incidence of anastomosis under tension, a situation more likely to result in a definitive failure. It is our clinical belief that it is the combination of extended length of the resected airway, loss of laryngeal stability, and supralaryngeal release maneuvers that favors the onset of deglutition disturbances and that additional release maneuvers (ie, hilar release) do not reduce the anastomotic tension to the extent that would justify a full vertical sternotomy. Moreover, pulling down the larynx is by far more physiologic than bringing up the intrathorax airway.

Sixth, a few patients may present with a TEF as well. Operations should not be denied for these patients after an adequate medical preoperative preparation. The specific surgical problem encountered by these patients is the relatively high site of the fistula, the repair of which may cause swallowing problems because of the vicinity of the upper esophageal sphincter.

In conclusion, the results demonstrate that the presented partial cricoidectomy with primary thyrotracheal anastomosis can be applied in patients with postintubation subglottis stenosis extending up to $1 \mathrm{~cm}$ below the vocal cords and measuring up to $6 \mathrm{~cm}$ in length with excellent-to-good results. The presence of a TEF does not contraindicate concomitant repair.

\section{REFERENCES}

1. Grillo HC, Mathisen DJ, Wain JC. Laryngotracheal resection and reconstruction for subglottic stenosis. Ann Thorac Surg 1992;153:54-63.

2. Pearson FG, Cooper JD, Nelems JM, Van Nonstrand AWP. Primary tracheal anastomosis after resection of the cricoid cartilage with preservation of the recurrent laryngeal nerves. J Thorac Cardiovasc Surg 1975;70:806-16.

3. Couraud L, Brichon PY, Velly JF. The surgical management of inflammatory and fibrous laryngotracheal stenosis. Eur J Cardiothorac Surg 1988;2:410-5.

4. Monnier P, Lang F, Savary M. Cricotracheal resection for pediatric subglottic stenosis. Int J Pediatr Otorhinolaryngol 1999;49:S283-6.

5. Macchiarini P, Chapelier A, Lenot B, Cerrina J, Dartevelle P. Laryngotracheal resection and reconstruction for post-intubation subglottic stenosis. Eur J Cardiothorac Surg 1993;7:300-5.

6. Rethi A. An operation for cicatricial stenosis of the larynx. J Laryngol Otol 1956;70:283-9.

7. Macchiarini P, Verhoye P, Chapelier A, Fadel E, Dartevelle P. Evaluation and outcome of different surgical techniques for postintubation tracheoesophageal fistula. J Thorac Cardiovasc Surg 2000;119:268-76.

8. Gerwat J, Bryce DP. The management of subglottic stenosis by resection and direct anastomosis. Laryngoscope 1974;84:940-7.

9. Conley JJ. Reconstruction of the subglottic air passage. Ann Otol Rhinol Laryngol 1953;62:477-95.

10. Ogura JH, Roper CL. Surgical correction of traumatic stenosis of the larynx and pharynx. Laryngoscope 1972;72:468-70.

11. Maddaus MA, Toth JL, Gullane PJ, Pearson FG. Subglottic tracheal resection and synchronous laryngeal reconstruction. J Thorac Cardiovasc Surg 1992;104:1443-50.

12. Pearson FG, Gullane P. Subglottic resection with primary tracheal anastomosis including synchronous laryngotracheal reconstruction. Semin Thorac Cardiovasc Surg 1996;4:381-91.

13. Couraud L, Jougon JB, Velly JF. Surgical treatment of nontumoral stenosis of the upper airway. Ann Thorac Surg 1995;60:250-9.

\section{Discussion}

Dr F. Griffith Pearson (London, Ontario, Canada). I would like to ask a few questions and make a few comments regarding technical issues.

You identified 45 patients in an age group between 4 and 70 -odd years. What number of patients did you actually have in the pediatric group that were age 4 years or even 
younger? If some of those patients were under 1 or 2 years old, did you use any modification of surgical technique? Phillippe Monnier has described a successful experience in infants and children and has recommended some technical modifications, including division of the lower one third of the thyroid cartilage.

You stated you did not use a subperichondrial freeing of the cricoid arch. I acquired knowledge of subperichondrial exposure of the cricoid arch from watching Louis Couraud many years ago, and I still think that subperichondrial mobilization of the anterior arch has advantages. It is less bloody, and if you are not anastomosing directly to the inferior border of the thyroid cartilage, it provides some substantial tissue with which to secure the upper side of the anterior anastomosis.

I was interested in your report of 23 of the 45 patients undergoing a release operation. The mean length of your resections was somewhere around $3 \mathrm{~cm}$. I do not believe you require a release for these relatively short resections. I think the longest resection was $5 \mathrm{~cm}$, and you described having tension in spite of the release. Did you use neck flexion intraoperatively during the actual anastomosis? It can deceive you if you do not. Did you use some type of chin-chest suture to maintain neck flexion postoperatively? I raise this issue because you describe, very clearly, transient but significant complications with dysphagia and aspiration in the patients who underwent supralaryngeal or suprahyoid release.

I congratulate you and your colleagues on the successful outcomes in those patients with TEF. They were clearly described in the article. I agree with you that the type of closure described with a mucosal closure and a separate muscular closure precludes the requirement for placing any type of interposed muscle graft between the trachea and esophagus.

I have one final question. You extubated many of your patients early. I understand that very few were intubated beyond about 5 days, and about $10 \%$ of patients were extubated within the first postoperative week. None of the resections were less than $1 \mathrm{~cm}$ below the vocal cords. When you resect closer than $1 \mathrm{~cm}$ below the cords, patients are much more likely to have glottic edema. However, your extubation mode is early and is very highly successful in a majority of cases. Did you use perioperative steroids to try and reduce glottic edema? That was another of Louis Couraud's contributions to the management of some of these very high and difficult resections.

Dr Douglas J. Mathisen (Boston, Mass). I congratulate you and your colleagues on the results in this difficult group of patients. I have just a couple of points.
This is very similar to the technique that Dr Grillo has devised for this problem, with one exception, and that is the use of the running stitch. Would you elaborate a bit on how you keep the tension off of that posterior wall when you use a running stitch when you complete the rest of the anastomosis?

I also have an issue relating to steroids. Were any of the patients receiving steroids beforehand, and if so, at what level and dose? Also, did you run into any problems with those patients who were taking steroids?

Dr Macchiarini. Thank you very much, Dr Pearson and Dr Mathisen. I will try my best to answer the questions.

Concerning the first question, we had 2 pediatric patients, and in these cases we usually made a vertical split on the thyroid cartilage.

I agree with you that the free perichondrial plane and its elevation is greatly advantageous, and in fact, we used that not for postintubation stenosis but for other types of stenosis that go very, very high in the posterior larynx. Using that plane, we have a more solid way to safely perform the anastomosis.

To reduce tension intraoperatively, we deflate the pillow that is behind the shoulder; we flex the head; and we try to be very elastic with the patient. We have not used routine chin fixation for almost 10 years.

I agree with you that when repairing a TEF, you do not need the muscle interposition simply because you remove the airway but leave in place the esophagus, and therefore the anastomoses are not in contact at all between them. We use perioperatively only $500 \mathrm{mg}$ IU of methylprednisolone (SoluMedrol) to dry the vocal cords before extubation, and this is systematically done.

With regard to the question of Dr Mathisen, the way we do the posterior plane is quite similar to the way we do any type of tracheobronchial reconstruction. We use a posterior PDS 4-0 suture, which picks, in these cases specifically, the remnant of the healthy mucosa proximally and the uppermost trachea and place the sutures until the posterior 2 stumps are all taken. We then push the sutures. We have the head flexed and the pillow deflated, and then the 2 parts, the back part of the anastomosis, are kept together. We then separately secure the anastomosis on the lateral aspect with two independent PDS 4-0 sutures.

As far as I can remember, only 1 patient had very high doses of steroids because of Crohn disease. We try to reduce these doses, and usually succeed, but we do accept patients with steroid doses of less than 10 or $15 \mathrm{mg} / \mathrm{d}$. 